\title{
Progress on the nature of the QCD thermal transition as a function of quark flavors and masses
}

\author{
Francesca Cuteri* \\ Institut für Theoretische Physik - Goethe-Universität Frankfurt am Main \\ Max-von-Laue-Str. 1, 60438 Frankfurt am Main \\ E-mail: cuteri@th.physik.uni-frankfurt.de
}

\section{Owe Philipsen}

Institut für Theoretische Physik - Goethe-Universität Frankfurt am Main Max-von-Laue-Str. 1, 60438 Frankfurt am Main,

John von Neumann Institute for Computing (NIC) GSI

Planckstr. 1, 64291 Darmstadt, Germany

E-mail: philipsen@th.physik.uni-frankfurt.de

\section{Alessandro Sciarra}

Institut für Theoretische Physik - Goethe-Universität Frankfurt am Main Max-von-Laue-Str. 1, 60438 Frankfurt am Main

E-mail: sciarraeth.physik.uni-frankfurt.de

\begin{abstract}
We investigate to which extent we can exploit the dependence of the order of the chiral transition on the number of light degenerate flavors $N_{\mathrm{f}}$, re-interpreted as continuous parameter in the path integral formulation, as a means to perform a controlled chiral extrapolation and deduce the order of the transition for the case $N_{\mathrm{f}}=2$, which is still under debate.
\end{abstract}

The 36th Annual International Symposium on Lattice Field Theory -1 LATTICE2018 22-28 July, 2018

Michigan State University, East Lansing, Michigan, USA.

${ }^{*}$ Speaker. 


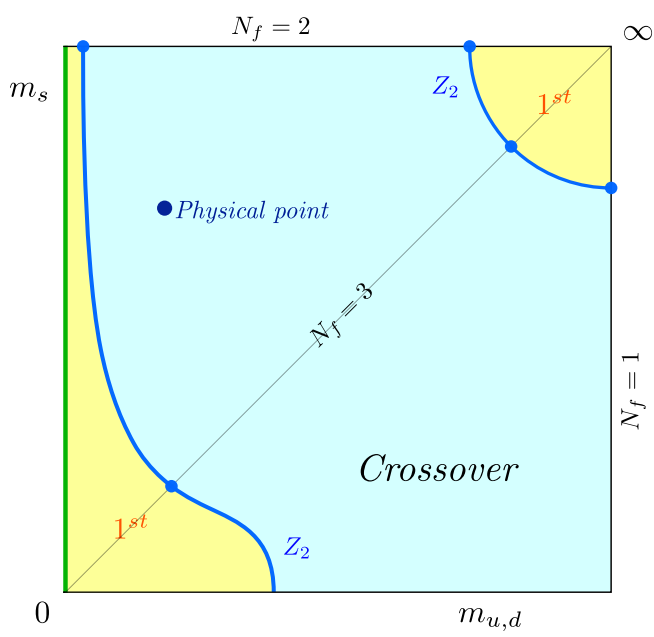

(a) First order scenario in the $m_{s}-m_{u, d}$ plane

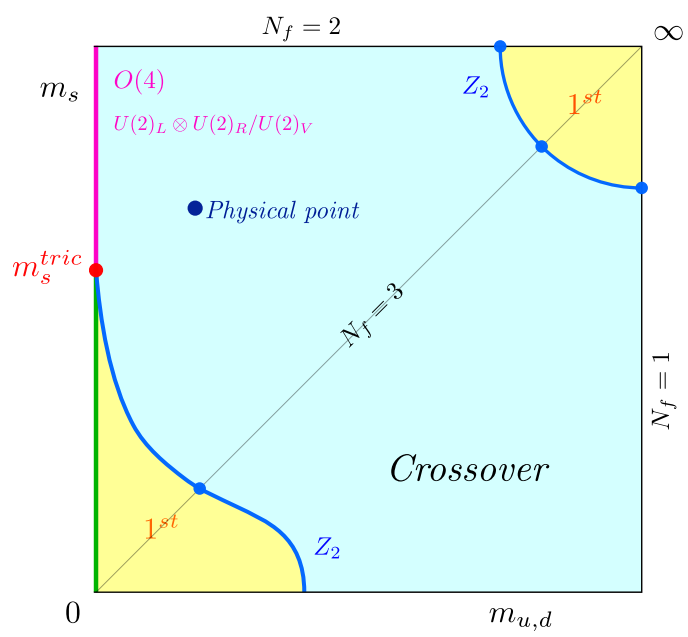

(b) Second order scenario in the $m_{s}-m_{u, d}$ plane.

Figure 1: Two possible scenarios for the order of the QCD thermal phase transition as function of the masses of quarks. Indicated in Fig. 1(b) are also plausible universality classes for the second order line at $m_{u, d}=0$.

\section{Introduction}

The Columbia plot, of which we show in Figure 1 two possible versions based on current findings, encapsulates our still very limited knowledge about the order of the thermal phase transition in QCD as function of the two light (assumed degenerate) quark masses $m_{u, d}$ and the strange quark mass $m_{s}$. Continuum extrapolated results are so far only available at the physical point. Elsewhere, using different unimproved [1-6] and improved [7-10] fermion discretizations, seemingly contradicting results have been obtained, in particular in what concerns the case of $N_{\mathrm{f}}=2,3$ degenerate light flavors in the limit of small masses corresponding to the top and bottom left corners in the Columbia plot, respectively.

This motivated us to push forward with studies aiming at elucidating, in particular, the picture for $N_{\mathrm{f}}=2$ degenerate light flavors, by exploiting the dependence of the chiral transition on the number of light degenerate flavors $N_{\mathrm{f}}$ as a means to perform controlled chiral extrapolations. To this end, we treated $N_{\mathrm{f}}$ as a continuous real parameter, of some statistical system behaving, at any integer $N_{\mathrm{f}}$ value, as QCD at zero density, with $N_{\mathrm{f}}$ mass-degenerate fermion species [11]

$$
Z_{N_{\mathrm{f}}}(m)=\int \mathscr{D} U[\operatorname{det} M(U, m)]^{N_{\mathrm{f}}} e^{-\mathscr{S}_{\mathrm{G}}} .
$$

Within this framework, the two considered scenarios for the Columbia plot can be put in oneto-one correspondence with the two sketches for the order of the thermal phase transition in the $\left(m, N_{\mathrm{f}}\right)$-plane displayed in Figure 2.

Our original strategy was to find out for which (tricritical) value $N_{\mathrm{f}}^{\text {tric }}$ the phase transition displayed by this system changes from first-order to second-order, by mapping out the $Z_{2}$ phase boundary. The extrapolation to the chiral limit with known tricritical exponents can then decide between the two scenarios, depending on whether $N_{\mathrm{f}}^{\text {tric }}$ is larger or smaller than 2 . 


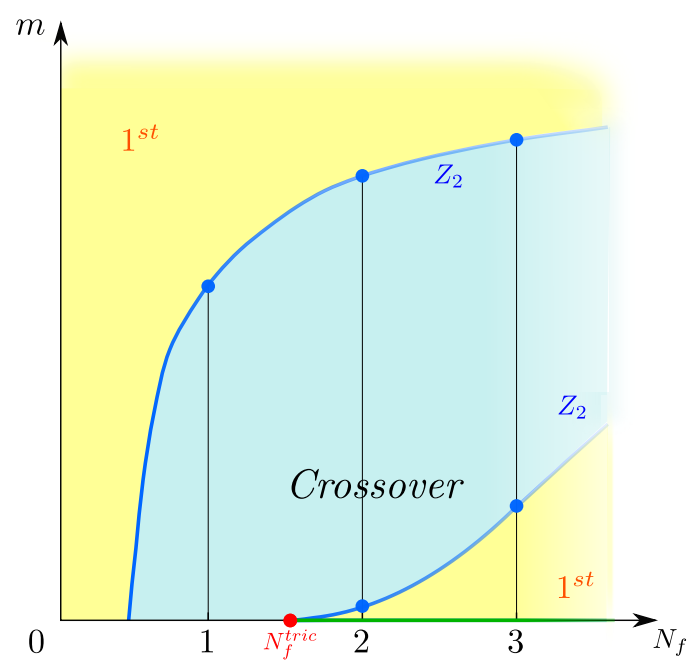

(a) First order scenario in the $m-N_{\mathrm{f}}$ plane

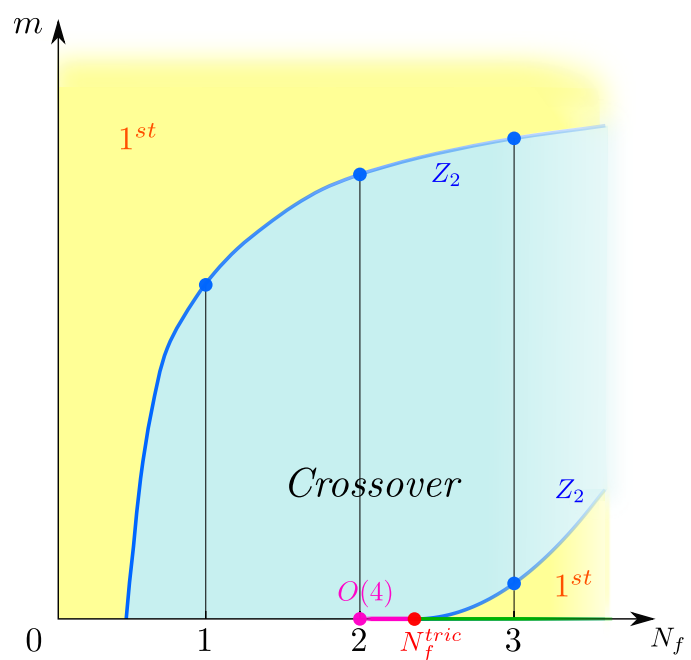

(b) Second order scenario in the $m-N_{\mathrm{f}}$ plane

Figure 2: The two considered possible scenarios for the order of the QCD thermal phase transition as function of the mass of the quarks and the number of degenerate fermion flavors.

While the tricritical scaling region was found to be very narrow already on coarse lattices, results at larger $m$ and $N_{\mathrm{f}}$ were found to feature, over a much wider region, a remarkable linear behavior, which was not expected on universality grounds.

What our findings suggest is that, if it is reasonable to expect both linearity within some range in $N_{\mathrm{f}}$ and tricritical scaling more in the chiral limit, then one would be able to make use of a linear extrapolation to $m=0$, to at least get $N_{\mathrm{f}}^{\text {lin }}$ as an upper bound for $N_{\mathrm{f}}^{\text {tric }}$, out of much more affordable simulations and possibly without even simulating at noninteger numbers of flavors.

For as long as the upper bound from the linear extrapolation keeps lying at $N_{\mathrm{f}}<2$, while one simulates at larger and larger $N_{\tau}$ values towards the continuum limit, one can infer that the transition in the $N_{\mathrm{f}}=2$ chiral limit is of first order. However, should our

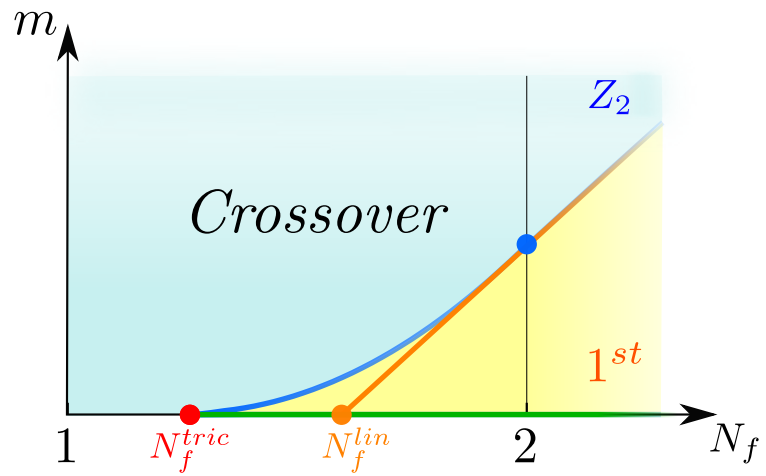

Figure 3: Sketch showing how, via a linear extrapolation to the chiral limit, $N_{\mathrm{f}}^{\text {lin }}$ as an upper bound for $N_{\mathrm{f}}^{\text {tric }}$ can be extracted, with the first order scenario being realized for as long as $N_{\mathrm{f}}^{\text {lin }}<2$. linear extrapolation give $N_{\mathrm{f}}^{\text {lin }} \gtrsim 2$, then knowledge of the size of the scaling region is necessary to draw conclusions.

\section{Numerical strategy}

We employ unimproved staggered fermions and use the RHMC algorithm [12] to simulate any number $N_{\mathrm{f}}$ of degenerate flavors, with $\frac{N_{\mathrm{f}}}{4}$ being the power to which the fermion determinant is 

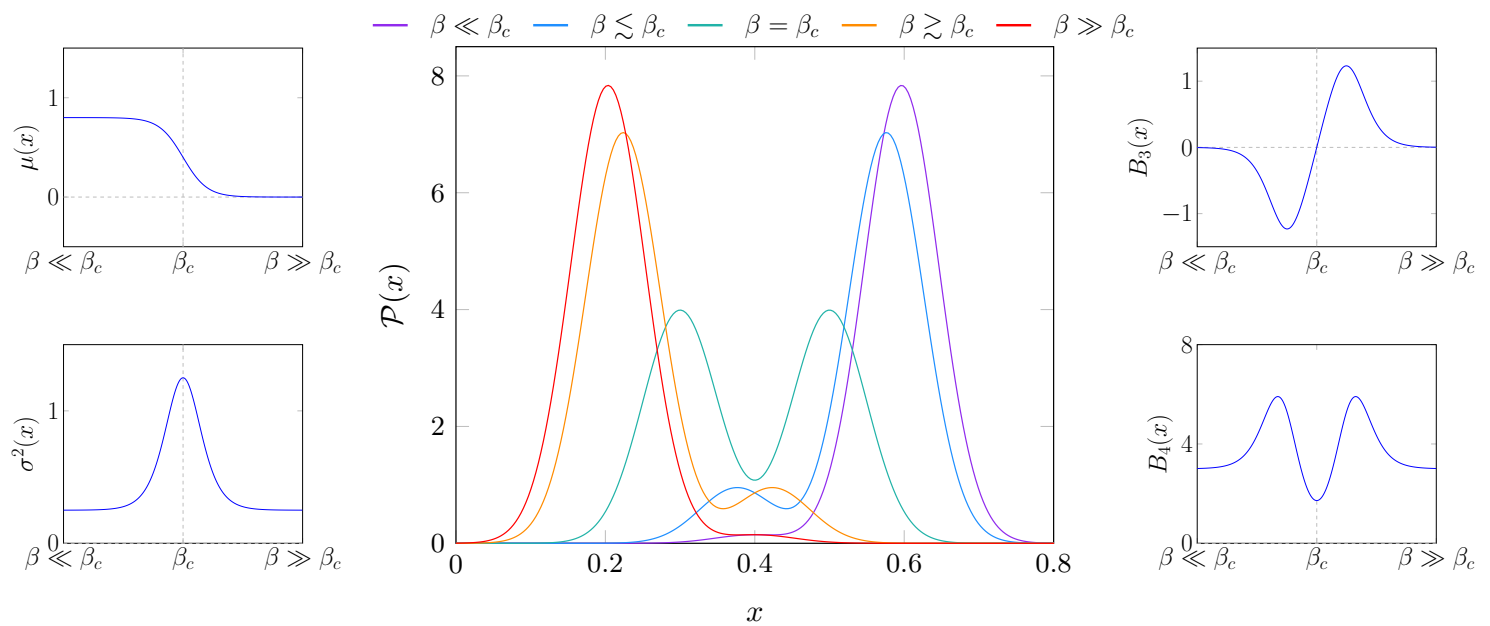

Figure 4: The chiral condensate distribution according to a model $\mathscr{P}(x)$ based on our numerical findings at various $\beta$ values and the corresponding moments as function of $\beta$. Details on the model are discussed in [11].

raised in $Z_{N_{\mathrm{f}}}(m)$. All numerical simulations are performed using the publicly available OpenCLbased code $C^{2} \mathrm{QCD}$ [13] of which a version 1.0 has been recently released [14]. We consider temporal extents $N_{\tau}=4,6$ to check for the cutoff dependence of $N_{\mathrm{f}}^{\text {lin }}$. The ranges in mass $m$ and gauge coupling constant $\beta$ of the investigated parameter space are dictated by our purpose of locating the chiral phase transition for values of the mass $m$ around the critical $m_{Z_{2}}$ value, with the temperature related to the coupling according to $T=1 /\left(a(\beta) N_{\tau}\right)$.

To locate and identify the order of the chiral phase transition we rely on a finite size scaling analysis of the third and fourth standardized moments of the distribution of the (approximate) order parameter. The $\mathrm{n}^{\text {th }}$ standardized moment for a generic observable $\mathscr{O}$ is expressed as

$$
B_{n}\left(\beta, m, N_{\sigma}\right)=\frac{\left\langle(\mathscr{O}-\langle\mathscr{O}\rangle)^{n}\right\rangle}{\left\langle(\mathscr{O}-\langle\mathscr{O}\rangle)^{2}\right\rangle^{n / 2}} .
$$

Being interested in the order of the thermal phase transition in the chiral limit, we consider the kurtosis $B_{4}(\beta, m)$ [15] of the sampled $\langle\bar{\psi} \psi\rangle$ distribution, evaluated at the coupling $\beta_{c}$ for which $B_{3}\left(\beta=\beta_{c}, m, N_{\sigma}\right)=0$, i.e. on the phase boundary.

In the thermodynamic limit $N_{\sigma} \rightarrow \infty$, the kurtosis $B_{4}\left(\beta_{c}, m\right)$ takes the values of 1 for a first order transition and 3 for an analytic crossover, respectively, with a discontinuity when passing from a first order region to a crossover region via a second order point. For the $3 D$ Ising universality class, which is the relevant one for our case, the kurtosis takes the value 1.604 [16]. The discontinuous step function is smeared out to a smooth function as soon as a finite volume is considered. In the lattice box, the distribution of the approximate order parameter and its higher moments behave, depending on $\beta$, as illustrated in Figure 4. Moreover, in the vicinity of a critical point, the kurtosis $B_{4}\left(\beta_{c}, m, N_{\sigma}\right)$ can be expanded in powers of the scaling variable $x \equiv\left(m-m_{Z_{2}}\right) N_{\sigma}^{1 / v}$, and, for large enough volumes, the expansion can be truncated after the linear term,

$$
B_{4}\left(\beta_{c}, m, N_{\sigma}\right) \simeq B_{4}\left(\beta_{c}, m_{Z_{2}}, \infty\right)+c\left(m-m_{Z_{2}}\right) N_{\sigma}^{1 / v} .
$$




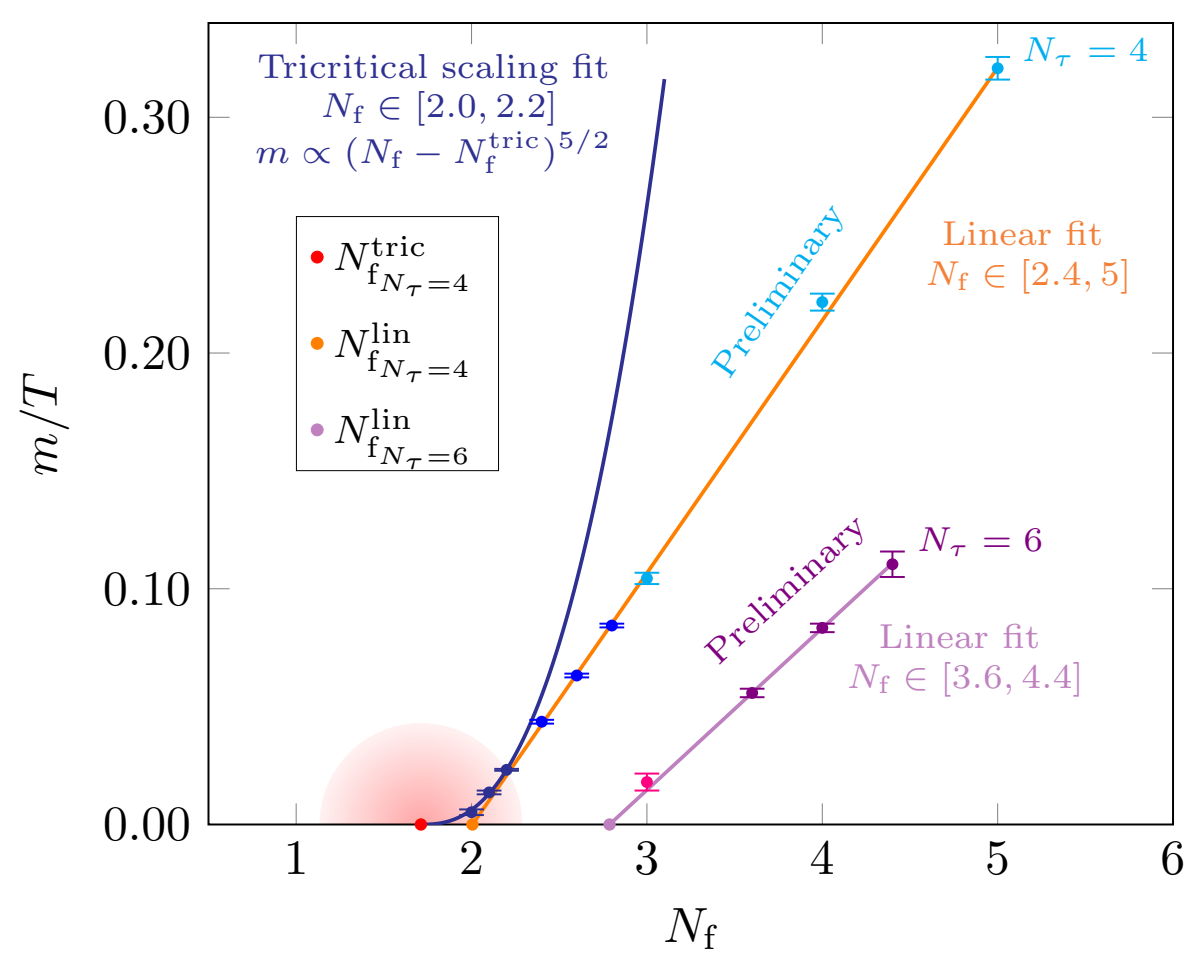

Figure 5: The $Z_{2}$ boundary in the $m / T-N_{\mathrm{f}}$ plane for $N_{\tau}=4,6$. The dark blue line represents the tricritical extrapolation to the chiral limit as in [11]. The orange line represents a linear extrapolation based on $m_{Z_{2}}$ in the $N_{\mathrm{f}}$ range 2.4-5 using also newly simulated points. The violet line represents a linear extrapolation on the basis of $m_{Z_{2}}$ in the $N_{\mathrm{f}}$ range 3.6-4.4. The magenta point at $N_{\tau}=6$ and $N_{\mathrm{f}}=3$ is borrowed from [3].

As already mentioned, in our case, the critical value for the mass $m_{Z_{2}}$ is known to correspond to a second order phase transition in the 3D Ising universality class, so we fix $B_{4}\left(\beta_{c}, m_{Z_{2}}, \infty\right)=1.604$ and $v=0.6301$ to better constrain the fit.

Our simulated values for $B_{4}\left(\beta_{c}, m, N_{\sigma}\right)$ are then fitted to Eq. (2.2) and the fit parameters $c$ and $m_{Z_{2}}$ are extracted. The whole study has been repeated for $N_{\mathrm{f}} \in\{3,4,5\}$ at $N_{\tau}=4$ and $N_{\mathrm{f}} \in\{3.6,4.0,4.4\}$ at $N_{\tau}=6$.

\section{Results and conclusions}

Our results are reported in Figure 5. The first important thing to observe is that, while tricritical extrapolation for $N_{\tau}=4$ resulted in $N_{\mathrm{f}}^{\text {tric }}<2$, providing a confirmation for the first order scenario being realized on coarse lattices, a linear extrapolation to the chiral limit using those data which exhibit linear scaling within the range $N_{\mathrm{f}} \in[2.4,5.0]$, results in $N_{\mathrm{f}}^{\text {lin }}=2$ within errors. Strictly speaking, by just considering $N_{\tau}=4$ results, one would conclude that the linear extrapolation alone cannot give conclusive answers on the order of the $N_{\mathrm{f}}=2$ transition in the chiral limit. However, results on finer lattices were produced as well. On $N_{\tau}=6$, what we observe is that data within 
the range $N_{\mathrm{f}} \in[3.6,4.4]$ certainly do not fall in the tricritical scaling region, but they do exhibit linear scaling. Moreover, if we consider the result for $N_{\mathrm{f}}=3$ for the same discretization from the literature [3], we can see it is fully consistent with our linear extrapolation. Finally, the most important aspect of this result is that, linearly extrapolating at $N_{\tau}=6$, we get $N_{\mathrm{f}}^{\text {lin }} \lesssim 3$, namely quite far to the right of $N_{\mathrm{f}}=2$.

To conclude, we have proposed and tested an approach, to clarify the order of the thermal transition in the chiral limit of QCD at zero chemical potential with two dynamical flavors of quarks. Specifically, a controlled chiral extrapolation in the $m-N_{f}$ plane with $N_{f}$ promoted to a continuous parameter in the path integral formulation of the theory is possible, given that if the transition for $m \rightarrow 0$ changes with $N_{f}$ from $1^{\text {st }}$ order (triple) to $2^{\text {nd }}$ by reducing $N_{\mathrm{f}}$, there has to exist a tricritical point at some $N_{\mathrm{f}}^{\text {tric }}$. Moreover, the linearity featured by the $Z_{2}$ boundary over a wide $N_{\mathrm{f}}$ region suggests that a linear extrapolation to $m=0$ can also provide an upper bound $N_{\mathrm{f}}^{\text {lin }}$ for $N_{\mathrm{f}}^{\text {tric }}$, which may become useful to discriminate between first and second order scenario and help resolving the " $N_{\mathrm{f}}=2$ puzzle".

Based on our numerical findings, the shift in the $Z_{2}$ critical boundary from $N_{\tau}=4$ to $N_{\tau}=6$ points towards a behavior consistent with that from improved actions on sufficiently fine lattices.

\section{Acknowledgements}

The authors acknowledge support by the Deutsche Forschungsgemeinschaft (DFG) through the grant CRC-TR 211 "Strong-interaction matter under extreme conditions" and by the Helmholtz International Center for FAIR within the LOEWE program of the State of Hesse. The project received initial support by the German BMBF under contract no. 05P1RFCA1/05P2015 (BMBFFSP 202). We also thank the staff of LOEWE-CSC and L-CSC for their support.

\section{References}

[1] F. Karsch, E. Laermann and C. Schmidt, The Chiral critical point in three-flavor QCD, Phys. Lett. B520 (2001) 41 [hep-lat/0107020].

[2] P. de Forcrand and O. Philipsen, The QCD phase diagram for three degenerate flavors and small baryon density, Nucl. Phys. B673 (2003) 170 [hep-lat/ 0307020 ].

[3] P. de Forcrand, S. Kim and O. Philipsen, A QCD chiral critical point at small chemical potential: Is it there or not?, PoS LAT2007 (2007) 178 [0711. 0262].

[4] C. Bonati, P. de Forcrand, M. D’Elia, O. Philipsen and F. Sanfilippo, Chiral phase transition in two-flavor QCD from an imaginary chemical potential, Phys. Rev. D90 (2014) 074030 [1408.5086].

[5] O. Philipsen and C. Pinke, The $N_{f}=2$ QCD chiral phase transition with Wilson fermions at zero and imaginary chemical potential, Phys. Rev. D93 (2016) 114507 [1602.06129].

[6] P. de Forcrand and M. D'Elia, Continuum limit and universality of the Columbia plot, PoS LATTICE2016 (2017) 081 [1702.00330].

[7] TMFT collaboration, F. Burger, E.-M. Ilgenfritz, M. Kirchner, M. P. Lombardo, M. Müller-Preussker, O. Philipsen et al., Thermal QCD transition with two flavors of twisted mass fermions, Phys. Rev. D87 (2013) 074508 [1102.4530]. 
[8] B. B. Brandt, A. Francis, H. B. Meyer, O. Philipsen, D. Robaina and H. Wittig, On the strength of the $U_{A}(1)$ anomaly at the chiral phase transition in $N_{f}=2$ QCD, JHEP 12 (2016) 158 [1608.06882].

[9] X.-Y. Jin, Y. Kuramashi, Y. Nakamura, S. Takeda and A. Ukawa, Critical point phase transition for finite temperature 3-flavor QCD with non-perturbatively $O(a)$ improved Wilson fermions at $N_{\mathrm{t}}=10$, Phys. Rev. D96 (2017) 034523 [1706.01178].

[10] A. Bazavov, H. T. Ding, P. Hegde, F. Karsch, E. Laermann, S. Mukherjee et al., Chiral phase structure of three flavor QCD at vanishing baryon number density, Phys. Rev. D95 (2017) 074505 [1701.03548].

[11] F. Cuteri, O. Philipsen and A. Sciarra, QCD chiral phase transition from noninteger numbers of flavors, Phys. Rev. D97 (2018) 114511 [1711.05658].

[12] A. D. Kennedy, I. Horvath and S. Sint, A New exact method for dynamical fermion computations with nonlocal actions, Nucl. Phys. Proc. Suppl. 73 (1999) 834 [hep-lat/9809092].

[13] O. Philipsen, C. Pinke, A. Sciarra and M. Bach, $C L^{2} Q C D$ - Lattice QCD based on OpenCL, PoS LAT2014 (2014) 038 [1411.5219].

[14] M. Bach, F. Cuteri, C. Czaban, C. Pinke, A. Sciarra et al., "CL2QCD: lattice QCD using OpenCL." CL2QCD - v1.0, since 2011.

[15] K. Binder, Finite size scaling analysis of Ising model block distribution functions, Z.Phys. B43 (1981) 119.

[16] A. Pelissetto and E. Vicari, Critical phenomena and renormalization group theory, Phys. Rept. 368 (2002) 549 [cond-mat/ 0012164$]$. 